\title{
Intrafractional 6D head movement increases with time of mask fixation during stereotactic intracranial RT-sessions
}

\author{
Julian Mangesius ${ }^{1}$, Thomas Seppi ${ }^{1}$, Rocco Weigel ${ }^{1}$, Christoph Reinhold Arnold', Danijela Vasiljevic ${ }^{1 *}$, \\ Georg Goebel' ${ }^{2}$ Peter Lukas', Ute Ganswindt ${ }^{1}$ and Meinhard Nevinny-Stickel ${ }^{1}$
}

\begin{abstract}
Background: The present study investigates the intrafractional accuracy of a frameless thermoplastic mask used for head immobilization during stereotactic radiotherapy. Non-invasive masks cannot completely prohibit head movements. Previous studies attempted to estimate the magnitude of intrafractional inaccuracy by means of preand postfractional measurements only. However, this might not be sufficient to accurately map also intrafractional head movements.
\end{abstract}

Materials and methods: Intrafractional deviation of mask-fixed head positions was measured in five patients during a total of 94 fractions by means of close-meshed repeated ExacTrac measurements (every 1.4 min) conducted during the entire treatment session. A median of six (range: 4 to 11) measurements were recorded per fraction, delivering a dataset of 453 measurements.

Results: Random errors (SD) for the $x, y$ and $z$ axes were $0.27 \mathrm{~mm}, 0.29 \mathrm{~mm}$ and $0.29 \mathrm{~mm}$, respectively. Median 3D deviation was $0.29 \mathrm{~mm}$. Of all 3D intrafractional motions, 5.5 and $0.4 \%$ exceeded $1 \mathrm{~mm}$ and $2 \mathrm{~mm}$, respectively. A moderate correlation between treatment duration and mean 3D displacement was determined $\left(r_{\mathrm{s}}=0.45\right)$. Mean 3D deviation increased from $0.21 \mathrm{~mm}(\mathrm{SD}=0.26 \mathrm{~mm})$ in the first $2 \mathrm{~min}$ to a maximum of $0.53 \mathrm{~mm}(\mathrm{SD}=0.31 \mathrm{~mm})$ after 10 min of treatment time.

Conclusion: Pre- and post-treatment measurement is not sufficient to adequately determine the range of intrafractional head motion. Thermoplastic masks provide both reliable interfractional and intrafractional immobilization for image-guided stereotactic hypofractionated radiotherapy. Greater positioning accuracy may be obtained by reducing treatment duration ( $<6 \mathrm{~min}$ ) and applying intrafractional correction.

Trial registration: Clinicaltrials.gov, NCT03896555, Registered 01 April 2019 - retrospectively registered.

Keywords: Frameless stereotactic radiation therapy, Thermoplastic mask head fixation, Intrafractional accuracy, X-ray position monitoring, ExacTrac

\section{Background}

In recent years, advances in non-invasive patient immobilization as well as in image-guided radiation therapy (IGRT) have enabled the use of thermoplastic masks and hypofractionated radiotherapy for single brain metastases [1].

\footnotetext{
* Correspondence: Danijela.Vasiljevic@i-med.ac.at

${ }^{1}$ Department of Therapeutic Radiology and Oncology, Medical University of Innsbruck, Anichstraße 35, A-6020 Innsbruck, Austria

Full list of author information is available at the end of the article
}

The use of non-invasive thermoplastic masks allows for fractionated RT, thereby overcoming the main limitation of invasive head fixation [2, 3]. Several studies have shown that image guidance makes set-up and repositioning uncertainty with the non-invasive mask immobilization comparable to that of invasive stereotactic ring application [2, 4-6]. Nevertheless, this method may have less intrafractional accuracy due to the nonrigid construction, indirect immobilization of the skull, and unpredictable patient movement. Many studies reported this effect only by means of quantifying pre- and 
postfractional deviations of the patient's head by either CBCT (cone beam computed tomography) or ExacTrac $[2,4,7,8]$. However, real intrafractional movements cannot be mapped by measuring the position of the head only at the beginning and the end of treatment since this gives no information on possible head movements during the individual irradiation treatments. Larger deviations would need to be accounted for by increasing the PTV margins, thereby exponentially increasing the irradiated volume and the risk of complications, such as radionecrosis [9-11]. Especially, novel single-isocenter intracranial irradiation techniques for multiple metastases [12-14] demand for highest precision since even smallest-scale rotational deviations may lead to insufficient target coverage of more distant lesions.

The purpose of the present study was to evaluate the precision and reliability of mask fixation of the head during the entire duration of stereotactic RT sessions. In order to assess position accuracy not only at the beginning and the end of the sessions, we repeatedly mapped deviations of the head position in both translation and rotation, by concomitantly measuring intrafractional movement using the ExacTrac 6D X-Ray Positioning System (Brainlab AG, Munich, Germany). From the obtained data we evaluated the need to adjust safety margins around the gross tumor volume (GTV).

\section{Materials and methods}

\section{Patients and inclusion criteria}

Intrafractional variations were evaluated in a nonrandomized group of five patients (Additional file 1: Table S1) during $\mathrm{N}_{\mathrm{F}}=96$ treatment sessions with a total of $N=551$ ExacTrac measurements. ExacTrac imaging was used multiple times to monitor intrafractional movements of the head during beam-on time of single sessions. Intrafractional measurements were not used to correct patient's head position during the RT session. Corrections were performed only once up-front, as is standard practice at our clinics.

The study involved patients who had a single intracranial tumor or metastasis. Linear accelerator-based stereotactic image-guided radiotherapy was administered between November 2014 and September 2015. Two patients were treated with a hypofractionated regimen (five fractions), whereas three patients were treated according to a conventionally fractionated schedule (30 fractions). Immobilization was performed with the Brainlab Thermoplastic Mask (Brainlab AG, Munich, Germany). To ensure patient compliance and provide a homogeneous study population all prospectively selected patients were required to have a Karnofsky Performance Score (KPS) greater than $80 \%$ as well as good cooperation capability. Treatment planning and course were identical for conventional and hypofractionated treatments (LinAc Versa HD, Elekta AB, Stockholm, Sweden). Target volume definition was performed on fused planning $\mathrm{CT}$ and contrast enhanced $\mathrm{t} 1$ weighted MRI images using Brainlab iPlan RT Image (v4.5.3; Brainlab AG, Munich, Germany). Treatment planning was performed with Brainlab iPlan RT Dose (v4.5.3) as well as Pinnacle (v9.8; Philips Medicals, Fitchburg, WI, USA).

\section{Clinical workflow and intrafractional measurements}

To detect intrafractional motion during treatment delivery, the ExacTrac in-room based monitoring system (Brainlab AG, Munich, Germany) was used as previously described $[15,16]$. It was employed in this study to repeatedly record $3 \mathrm{D}$ deviations of the target isocenter for both translation and rotation, during a session of dose application that lasted up to 20 min (workflow shown in Additional file 1:Figure S1).

Following thermoplastic mask molding, contrastenhanced treatment planning CT was performed with a reconstructed slice thickness of $1.5 \mathrm{~mm}$. CT scans were also used for image registration to reference ExacTrac recordings and $\mathrm{CBCT}$-guided patient positioning at the beginning of each treatment session.

Calculated 6D shifts were checked and, if indicated, translational and rotational deviations from reference positions were computed and corrected by adjusting the treatment couch (equipped with the HexaPod evo RT system, Elekta AB, Stockholm, Sweden). CBCT check was repeated until translational deviation in each direction was $<1.0 \mathrm{~mm}$ and rotational errors were $<1.0^{\circ}$. Next, the first ExacTrac measurement was taken before treatment start at a rotatable baseplate position of $0^{\circ}$. This initial ExacTrac recording was used as a reference point for comparison with the subsequent intrafractional measurements made during irradiation $(N=3$ to 10$)$. Patient positions were not corrected during a treatment session.

Treatment plans in this study comprised both converging arcs with conical collimators and multiple isocentric fields with individually shaped beams using micro-multileaf collimator. ExacTrac measurements were taken simultaneously with arc irradiations (five per fraction) at gantry angles of $0^{\circ}, 90^{\circ}, 180^{\circ}$ or $270^{\circ}$ with a tolerance range of $+/-10^{\circ}$. For shaped beam application, ExacTrac recordings were taken immediately after each field application (five to eight per fraction). At baseplate angles of $90^{\circ}$ and $270^{\circ}$ it was not possible to detect positioning since the couch-mounted metal frame used for mask fixation shields parts of the ExacTrac radiographs. A final pair of ExacTrac images at a reset baseplate position of $0^{\circ}$ was acquired at the end of each treatment session. 


\section{Correction of data and phantom study}

At each step of the radiotherapy treatment course different sources of inaccuracy can accumulate. To correctly map the patient's intrafractional motions depending on mask fixation, other factors potentially causing positioning errors had to be eliminated. Ideally, the linear accelerator's gantry, collimator, and table all rotate with respect to a single point called the isocenter. In reality, unavoidable misalignments prevent the rotation axes from intersecting at a single point, but instead only near each other within a sphere [17].

The baseplate angle position was identified as a main system-inherent source of measured isocenter deviations. The deviation was largest at an angle of $50^{\circ}$ with an average 3D vector (3DV) of $0.82 \mathrm{~mm}$. Phantom measurements were performed to quantify this error for each baseplate angle used in this study in order to correct our results by considering the recorded deviations.

A second cause of mask-independent errors can be addressed to residual errors derived from initial patient positioning. The first ExacTrac measurement of every fraction was therefore used as a point of reference and mask-independent 6D deviations were subtracted from all subsequent measurements.

\section{Statistical analysis}

Translations in the $\mathrm{x}$ (medial-lateral), $\mathrm{y}$ (superior-inferior) and $\mathrm{z}$ (anterior-posterior) directions as well as rotations around the $\mathrm{x}$ (transversal), $\mathrm{y}$ (longitudinal) and $\mathrm{z}$ (sagittal) axes were analysed. The resulting 3DV was calculated as follows:

$$
3 D V=\sqrt{x^{2}+y^{2}+z^{2}}
$$

IBM SPSS Statistics 22 (IBM Cooperation, Armonk, NY, USA) was used for statistical analysis of corrected values. An independent samples $t$-test was performed to compare positioning deviations of the first five fractions with those of the subsequent ones in the group of conventionally fractionated patients. To evaluate the correlation between the time elapsed since the first measurement in each treatment session and the 3DV, Spearman's rank correlation coefficient was calculated. An analysis of variance (ANOVA) was used to analyse this correlation further. A $p$ value of $<0.05$ was deemed significant.

\section{Results}

A total of 551 ExacTrac measurements were made with thermoplastic masks during 96 fractions of facilitated positioning. Two measurements had to be excluded from statistical analysis because of failed fusion between ExacTrac radiographs and DRRs. The first measurement of each fraction was used as reference. Hence, a total of 453 positioning recordings were statistically analysed.

The mean number of measurements per fraction was six and ranged from four to 11 . The recorded translational and rotational errors are presented in Table 1. Standard deviation (SD) of spatial displacements, used as a measure of random errors, was $0.27 \mathrm{~mm}, 0.29 \mathrm{~mm}$ and $0.29 \mathrm{~mm}$ in the $\mathrm{x}, \mathrm{y}$ and $\mathrm{z}$ directions, respectively. SD of the corresponding rotational errors was $0.33^{\circ}, 0.28^{\circ}$ and $0.55^{\circ}$. Maximum spatial displacements (Fig. 1) were 1.74 $\mathrm{mm}$ ( $\mathrm{x}$ axis), $1.49 \mathrm{~mm}$ (y axis) and $2.18 \mathrm{~mm}$ ( $\mathrm{z}$ axis), and the largest rotational errors were $2.18^{\circ}$ ( $\mathrm{x}^{\circ}$ angle), $1.52^{\circ}$ ( $y^{\circ}$ angle), and $2.27^{\circ}\left(\mathrm{z}^{\circ}\right.$ angle).

Mean deviation along the individual axes was close to the point of origin $(<0.10 \mathrm{~mm})$, accompanied by an average rotation angle of $<0.08^{\circ}$ for each axis. As a result, no significant systematic deviation was recorded. In absolute values, $95 \%$ of the deviations were smaller than 0.56 $\mathrm{mm}(\mathrm{x}), 0.62 \mathrm{~mm}(\mathrm{y})$ and $0.63 \mathrm{~mm}(\mathrm{z})$. Mean 3DV deviation was $0.38 \mathrm{~mm}(\mathrm{SD}=0.34 \mathrm{~mm}$; upper $95 \% \mathrm{CI}=0.41$ $\mathrm{mm})$. Of all 3D intrafractional motions $18.5,5.5$ and $0.4 \%$ exceeded $0.5 \mathrm{~mm}, 1 \mathrm{~mm}$ and $2 \mathrm{~mm}$, respectively. A maximum 3DV error of $2.82 \mathrm{~mm}$ was recorded. Of the 3DV deviations 95\% were smaller than $1.06 \mathrm{~mm}$ (Fig. 2).

Independent samples $t$-test delivered no difference $(\mathrm{t}=1.239, \mathrm{df}=391, p=0.216)$ in intrafractional motion of conventionally fractionated patients between the first five (mean $3 \mathrm{DV}=0.37 \mathrm{~mm}, \mathrm{SD}=0.32 \mathrm{~mm}$ ) and the remaining 25 treatment sessions (mean $3 \mathrm{DV}=0.32 \mathrm{~mm}$, $\mathrm{SD}=0.28 \mathrm{~mm}$ ). Mean duration from the first to the last ExacTrac measurement in each treatment session was 9 $\min 18 \mathrm{~s}\left(\mathrm{~N}_{\mathrm{F}}=96 ;\right.$ Min. $=4 \min 29 \mathrm{~s} ;$ Max. $=19 \min 36$ s). A moderate correlation between head motion (3DV) and time elapsed since the first measurement in each session was observed (Fig. 3). Spearman's rank

Table 1 Overview of translational and rotational errors with descriptive statistics of absolute deviations in head position caused by patient motion

\begin{tabular}{|c|c|c|c|c|c|c|c|}
\hline \multirow{2}{*}{$\begin{array}{l}N=453 \\
\left(N_{F}=96\right)\end{array}$} & \multirow{2}{*}{$\begin{array}{l}3 \mathrm{DV} \\
{[\mathrm{mm}]}\end{array}$} & \multicolumn{3}{|c|}{ Translations [mm] } & \multicolumn{3}{|c|}{ Rotations [ $\left.{ }^{\circ}\right]$} \\
\hline & & $\bar{x}$ & $Y$ & Z & $\overline{x^{\circ}}$ & $Y^{\circ}$ & $Z^{\circ}$ \\
\hline Median & 0.29 & 0.11 & 0.12 & 0.14 & 0.15 & 0.11 & 0.22 \\
\hline Mean & 0.38 & 0.17 & 0.19 & 0.21 & 0.22 & 0.19 & 0.36 \\
\hline \multicolumn{8}{|l|}{$95 \% \mathrm{Cl}$} \\
\hline Lower & 0.35 & 0.15 & 0.17 & 0.19 & 0.19 & 0.17 & 0.32 \\
\hline Upper & 0.41 & 0.19 & 0.21 & 0.23 & 0.24 & 0.21 & 0.40 \\
\hline Variance & 0.11 & 0.05 & 0.05 & 0.05 & 0.06 & 0.04 & 0.17 \\
\hline SD & 0.34 & 0.22 & 0.22 & 0.23 & 0.25 & 0.21 & 0.42 \\
\hline Maximum & 2.82 & 1.74 & 1.49 & 2.18 & 2.81 & 1.52 & 4.13 \\
\hline \multicolumn{8}{|l|}{ Percentile } \\
\hline $95 \%$ & 1.06 & 0.56 & 0.62 & 0.63 & 0.62 & 0.58 & 1.14 \\
\hline $25 \%$ & 0.16 & 0.04 & 0.05 & 0.06 & 0.07 & 0.05 & 0.09 \\
\hline
\end{tabular}



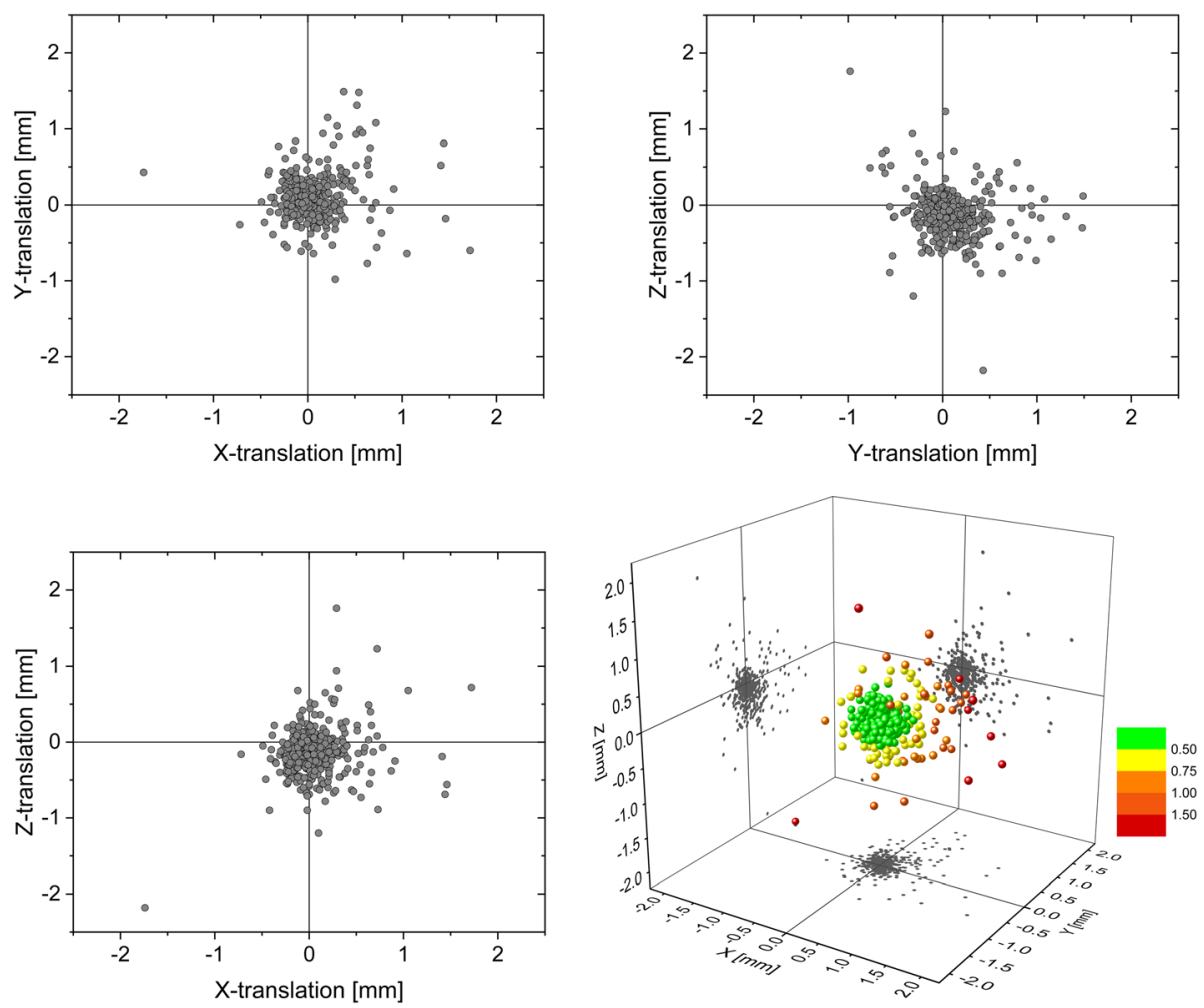

Fig. 1 Scatterplots of intrafractional positioning deviations in the coronal plane (xy), axial plane (xz) and sagittal plane (yz) during thermoplastic mask fixation of the head

correlation was run to analyse this relationship, which was statistically significant $\left(\mathrm{r}_{\mathrm{s}}=0.45, N=453, p<0.01\right)$.

Data points for 3DV head displacements were split into groups of consecutive 2-min intervals (Fig. 4). Oneway ANOVA was run to determine whether significant differences can be observed in head motion depending on the duration of treatment. Grouped measurement

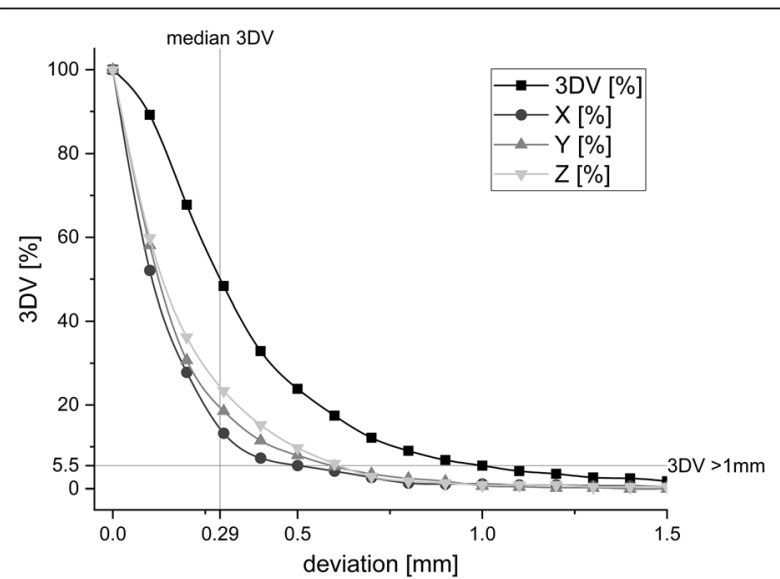

Fig. 2 Histogram of cumulative intrafractional 3DV displacements and proportionate $x$ (transversal), y (longitudinal) and $z$ (sagittal) translations using a thermoplastic mask for head fixation results used in this test are described in Additional file 1: Table S2. Significant differences between the extent of head displacements were observed between the 2-min interval groups $(\mathrm{F}(6,446)=9.790, p<0.001)$. A post hoc Games-Howell test showed a significant increase in quantified positioning deviations within the three

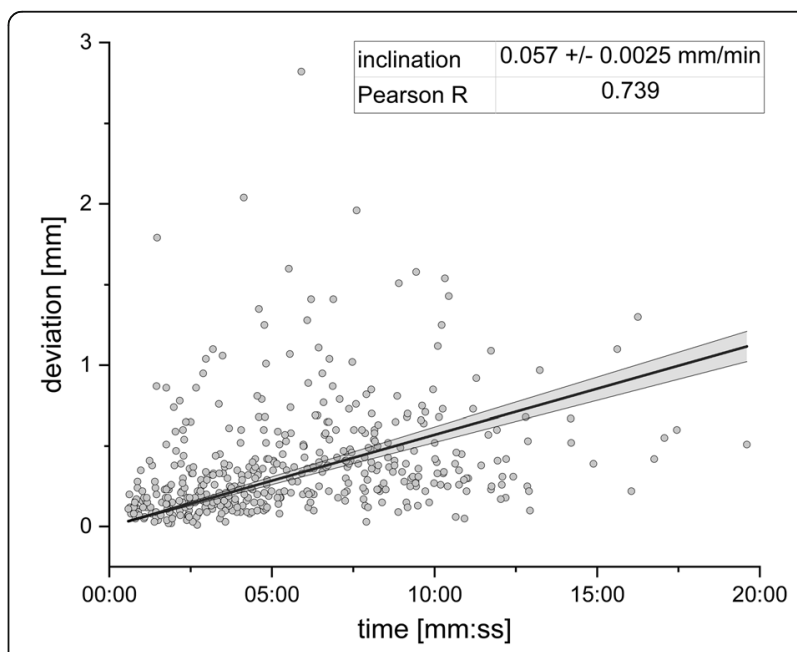

Fig. 3 Correlation between head motion (3DV) and elapsed time of intrafractional head fixation using a thermoplastic mask 

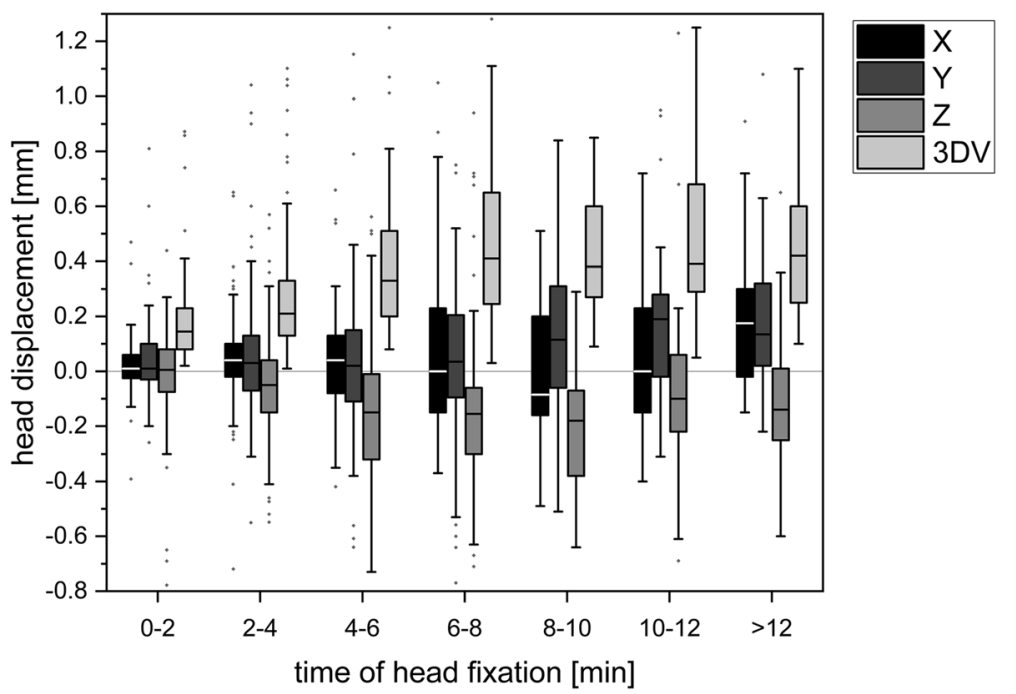

fead fixation [min]

Fig. 4 Head motion as a consequence of intrafractional treatment duration

interval groups of the first $6 \mathrm{~min}(p<0.03)$. No significant further increase in the spatial deviation of maskfixed head positions was observed after $8 \mathrm{~min}$. Mean 3DV head displacements increased from $0.21 \mathrm{~mm}(\mathrm{SD}=$ $0.26 \mathrm{~mm}$ ) in the 0 - to 2 -min interval group to a maximum of $0.53 \mathrm{~mm}(\mathrm{SD}=0.31 \mathrm{~mm})$ after $10 \mathrm{~min}$ of treatment time.

Time-dependent deviations were also analysed separately for each axis. One-way ANOVA showed a significant difference along the longitudinal $y$ axis ( $\mathrm{F}[6$, $446]=3.12, p=0.005$ ) and the sagittal $\mathrm{z}$ axis ( $\mathrm{F}[6$, $446]=3.51, p=0.002)$. Overall, discrete time-dependent systematic movements in the positive y direction and in the negative $\mathrm{z}$ direction were identified.

In addition, the three-dimensional variance in patient head position between two consecutive ExacTrac measurements $\left({ }_{\triangle} 3 \mathrm{DV}\right)$ during an intrafractional treatment course was quantified. A continuous 3D head motion between each measurement was observed and its absolute value in spatial deviation calculated (Fig. 5). Descriptive statistics are displayed in Table 2. A significant increase in intercourse positioning variance was demonstrated with one-way ANOVA $(\mathrm{F}(7,438)=8.30, p<$ 0.001). Mean magnitude of continuous intrafractional patient motions increased depending on the duration of treatment. Mean ${ }_{\triangle} 3 \mathrm{DV}$ assessed during the first $2 \mathrm{~min}$ of treatment was $0.21 \mathrm{~mm}(\mathrm{SD}=0.26 \mathrm{~mm})$ and reached $0.66 \mathrm{~mm}(\mathrm{SD}=0.41 \mathrm{~mm})$ after $12 \mathrm{~min}$.

To conclude, ExacTrac coordinates of maximum intrafractional head position deviation and the respective coordinates at the beginning and the end of fraction were compared. In 43 (45.7\%) of 96 analyzed fractions, 3D deviation did not exceed $0.5 \mathrm{~mm}$ - either during intrafractional measurements or at the end of the treatment session - as compared to the initial head position at treatment start. In 26 (27.7\%) of 94 fractions at least one intrafractional measurement showed a deviation larger than $0.5 \mathrm{~mm}$. However final ExacTrac measurement at the end of the session again revealed a 3D deviation of less than $0.5 \mathrm{~mm}$. Finally, in $18(19.1 \%)$ of 94 fractions, a deviation of more than $0.5 \mathrm{~mm}$ in mask-fixed head position was found both during the session and after completion of RT.

\section{Discussion}

The high radiation doses used and the proximity to vital structures make accurate positioning and precise irradiation of utmost importance in the radio-surgical treatment of intracranial metastases. The use of

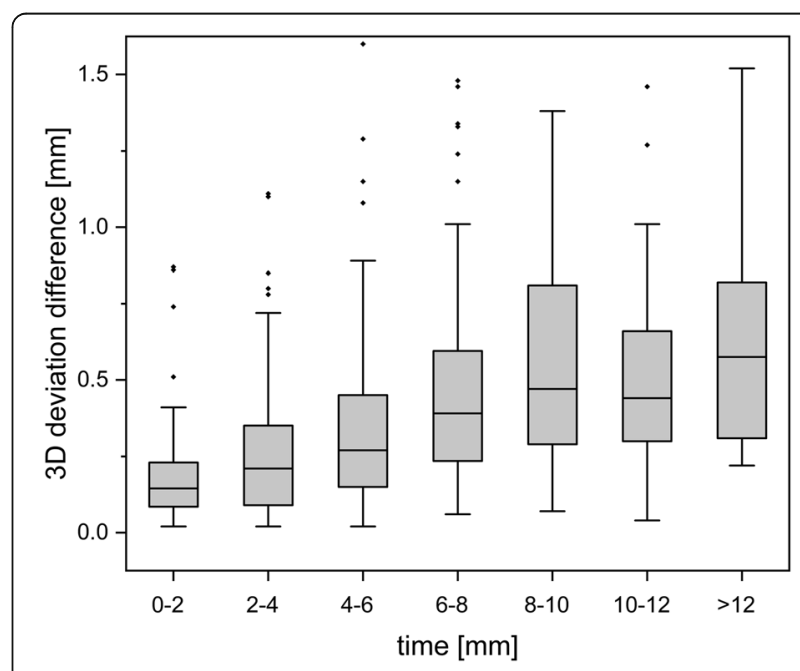

Fig. 5 Continuous 3D head motion between individual consecutive ExacTrac measurements ( $\triangle \mathrm{BDV}$ ) within a treatment session 
Table 2 Descriptive statistics of continuous 3D head motion between single consecutive ExacTrac measurements $(\triangle 3 D V)$ within a treatment session

\begin{tabular}{llllll}
\hline $\begin{array}{l}\text { Time } \\
{[\mathrm{min}]}\end{array}$ & $N$ & $\begin{array}{l}\Delta \text { 3DV } \\
\text { Mean } \\
{[\mathrm{mm}]}\end{array}$ & SD & & \multicolumn{2}{c}{ Confidence interval of the mean } \\
\cline { 6 - 6 } & & 0.21 & 0.26 & 0.15 & Upper \\
\hline $0-2$ & 68 & $0.29 \mathrm{n}$ & 0.27 \\
$2-4$ & 113 & 0.29 & 0.29 & 0.24 & 0.35 \\
$4-6$ & 87 & 0.39 & 0.42 & 0.30 & 0.48 \\
$6-8$ & 72 & 0.52 & 0.42 & 0.42 & 0.61 \\
$8-10$ & 58 & 0.61 & 0.53 & 0.47 & 0.75 \\
$10-12$ & 33 & 0.53 & 0.38 & 0.40 & 0.67 \\
$>12$ & 22 & 0.66 & 0.41 & 0.47 & 0.84 \\
\hline
\end{tabular}

thermoplastic masks offers substantial advantages concerning ease of use and patient comfort. In IGRT, the repositioning accuracy of thermoplastic masks, as compared to that of frame-based stereotactic head fixation, has been demonstrated in several studies to be equal $(<$ $1 \mathrm{~mm})[2,4,5,18]$.

Kataria et al. [19] investigated positioning accuracy of a thermoplastic mask using pre- and post-fractional imaging in six patients. The mask's mean displacements at the end of the treatment sessions were reported to be $0.60 \mathrm{~mm}(\mathrm{SD}=1.80 \mathrm{~mm}), 0.20 \mathrm{~mm}(\mathrm{SD}=0.60 \mathrm{~mm})$ and $0.00 \mathrm{~mm}(\mathrm{SD}=0.50 \mathrm{~mm})$ in the $\mathrm{x}, \mathrm{y}$ and $\mathrm{z}$ directions, respectively. In a similar study Ramakrishna et al. [2] reported data obtained from 110 stereotactic radiotherapy sessions and found a mean 3D deviation of $0.7 \mathrm{~mm}$ $(\mathrm{SD}=0.5 \mathrm{~mm})$. In $22 \%$ of all sessions a 3D displacement of larger than $1 \mathrm{~mm}$ was found. Linthout et al. [7] published data obtained from 385 pre- and postfractional stereoscopic $\mathrm{x}$-ray images. The mean translations were $0.0 \mathrm{~mm}(\mathrm{SD}=0.7 \mathrm{~mm}), 0.3 \mathrm{~mm}(\mathrm{SD}=0.7 \mathrm{~mm})$ and -0.5 $\mathrm{mm}(\mathrm{SD}=1.2 \mathrm{~mm})$ in the three directions, and mean rotational errors were $-0.2^{\circ} \quad\left(\mathrm{SD}=0.8^{\circ}\right), 0.1^{\circ} \quad\left(\mathrm{SD}=0.7^{\circ}\right)$ and $-0.1^{\circ} \quad\left(\mathrm{SD}=0.6^{\circ}\right)$. Lamba et al. [4] found translational deviations of $0.1 \mathrm{~mm}(\mathrm{SD}=0.3 \mathrm{~mm}),-0.1 \mathrm{~mm}$ $(\mathrm{SD}=0.5 \mathrm{~mm})$ and $0.1 \mathrm{~mm}(\mathrm{SD}=0.3 \mathrm{~mm})$. Of the measurements $6.5 \%$ exceeded $1 \mathrm{~mm}$ in any direction.

All of these studies measured the 'intra'fractional deviations by comparing only pre- and post-fractional measurements from CBCT or stereoscopic $\mathrm{x}$ ray imaging. However, this procedure is not suitable for determining the loss of accuracy caused by intrafractional head motions during an individual treatment session, because the magnitude, direction and angle of the motions do not necessarily remain constant over the course of treatment.

In the present study we measured the intrafractional accuracy by making repeated ExacTrac measurements per session in a non-randomized group of five patients delivering a dataset of 453 measurements recorded during 96 treatment sessions.
Random positioning errors assessed in our study show overall smaller intrafractional head displacements than in the previously described studies. Random errors (SD) in the translational deviations did not exceed 0.29 $\mathrm{mm}$ in any direction. The largest random rotational error (SD) was $0.55^{\circ}$. The mean 3DV length was 0.38 $\mathrm{mm}$. No relevant systematic deviation was observed. By contrast to others, only $5.5 \%$ of all $3 \mathrm{DV}$ measurements exceeded $1 \mathrm{~mm}$.

Accounting for only the first and last ExacTrac measurement of each investigated fraction in our study would result in an increase to $0.49 \mathrm{~mm}$ in calculated mean intrafractional 3D error $(+29.2 \%)$ versus the mean 3DV length of $0.38 \mathrm{~mm}$ determined from four to 11 measurements per session. Studies using only pre- and postfractional position measurements therefore tend to overestimate the influence of intrafractional motions on factual treatment accuracy impairment.

A possible explanation for these contradicting findings might be our finding that the magnitude of motion continuously increases with time during radiotherapy sessions. In addition, the time interval between pre- and postfractional measurements in the discussed studies was substantially longer ( $15 \mathrm{~min}$ to even exceeding 30 min in some cases) than in our study, which showed a mean time interval of repeated intrafractional measurements of $1.5 \mathrm{~min}$.

The relevance of real intrafractional head motion is further underlined by our finding that during $27.7 \%$ of all investigated fractions intrafractional motion of more than $0.5 \mathrm{~mm}$ was detected at least once during beam delivery, whereas the final ExacTrac measurement at the end of these sessions revealed a 3D deviation of less than $0.5 \mathrm{~mm}$.

Badakhshi et al. [20] examined intrafractional motions in a prospective cohort of patients during 269 stereotactic radiosurgery sessions using ExacTrac measurements after each new table position (3.6 measurements per fraction). Mean SD of all translations in any direction was reported as $0.8 \mathrm{~mm}$. Mean 3DV was $1.05 \mathrm{~mm}$ (SD = $0.93 \mathrm{~mm}$ ). Displacement errors were corrected when exceeding a value of $0.7 \mathrm{~mm}$ or $1^{\circ}$. Despite this measure, still $37 \%$ of all 3D deviations exceeded $1.0 \mathrm{~mm}$ as compared to $5.5 \%$ as reported by us. The timeframe between measurements $(\sim 4.2 \mathrm{~min})$ was also markedly longer than that applied in our study, thus potentially helping explain the substantial differences in positioning accuracy, even though the same mask system was used.

In contradiction to Lewis et al. [21], we detected a moderate correlation $\left(r_{s}=0.45\right)$ between time elapsed since setup and observed 3DV length. Within the first 6 min of treatment, a steep increase in 3D translational errors from $0.21 \mathrm{~mm}(\mathrm{SD}=0.26 \mathrm{~mm})$ to $0.51 \mathrm{~mm}(\mathrm{SD}=$ $0.35 \mathrm{~mm}$ ) was observed, reaching a plateau after 8 to 10 
min. These results are analogous to those reported by Amelio et al. [22], reflecting the influence of the time interval between pre- and postfractional measurements on intrafractional positioning accuracy. The authors assumed that patients might start to relax after a certain adaptation period, thereby causing the intrafractional displacements to reach a plateau.

However, this hypothesis contradicts our findings, which clearly show that the magnitude of individual intrafractional motions $\left({ }_{\triangle} 3 \mathrm{DV}\right)$ increased continuously along with each consecutive ExacTrac measurement during treatment. Although patient restlessness continuously increases during a treatment session, the thermoplastic mask's semi-flexible material restricts spatial displacements of the isocenter to a certain range until maximum freedom of movement has been reached.

This might explain why the magnitude of new movements still increases over time, without causing an increase in total positional displacement. Our finding is supported by Wang et al. [23], who also noted a timedependent increase in 3DV length from $0.34 \mathrm{~mm}$ to $0.77 \mathrm{~mm}$ within a time-frame of $45 \mathrm{~min}$ during 50 radiosurgery sessions. Along with our own results, decreasing the duration of RT sessions correlates with less intrafractional positional displacement, thus increasing overall intrafractional treatment accuracy.

The present study focused on the technical aspects of the feasibility to perform intrafractional X-ray based position monitoring, and aimed to evaluate the need for additional corrections during beam-on time of a single RT session. As such, the total number of anew patient positionings (96 fractions) as well as the number of position measurements $(n=453)$ is more decisive than the total number of patients. The small sample size is a limitation of this study and might impair the generalisability of our results. Therefore future investigations including a larger patient cohort will be necessary for statistically robust analysis of the frequency, time-course, and extent of intrafractional motions.

To minimise inter-patient variability the included patients were selected for good general condition and cooperation. In fact, the study was designed primarily to demonstrate the additional inaccuracy caused by intrafractional motions, excluding all other errors that might contribute to the determination of safety margins. Such error sources might differ between institutions, mainly depending on the applied RT and head fixation technique, as well as on available treatment devices. These errors include treatment machine related uncertainties, patient (re)positioning inaccuracy, imaging related limitations for treatment planning and for image guidance, as well as target definition and treatment planning system uncertainties [24]. The fact that $5 \%$ of measurements showed head displacements exceeding $1 \mathrm{~mm}$ - after exclusion of all other errors except for patient motion - justifies a more in-depth discussion on the necessity of intrafractional corrections. In addition, even the most cooperative patients selected for this study exhibited a time-dependent increase in head motion, finally surpassing tolerance after 6 to $8 \mathrm{~min}$ of uncorrected head fixation.

To summarise, measuring the positional variation, not only before but also during beam-on time of arc radiation therapy, allows determining the exact position error also during treatment. However, position corrections upon head displacements exceeding tolerance during the beam-on time were not performed in this study, since ExacTrac imaging had to be manually triggered, and 6D displacements were recorded for later analysis only. In order to benefit from the evidenced feasibility of intra-beam position surveillance, it would therefore be essential also to implement automated beam-hold, subsequent position correction, and precise resumption of LinAc-based irradiation.

\section{Conclusions}

Spatial displacements of the head during administration of stereotactic radiotherapy measured in this study for frameless head fixation are substantially smaller than reported by others. Safety margins of $1 \mathrm{~mm}$ were seen to be appropriate to account for at least $94.5 \%$ of 453 evaluations of intrafractional head positions in this highly selected group of five patients, confirming that thermoplastic masks provide adequately precise and reliable inter- and intrafractional immobilization for imageguided stereotactic radiotherapy.

To especially examine potential intrafractional head displacements, pre- and postfractional imaging alone is not suitable. For this purpose repeated intrafractional ExacTrac measurements were performed, showing that head motion increases depending on the duration of treatment. Consequently, greater intrafractional accuracy is achieved by reducing the duration of RT sessions. Alternatively, repeated verification of head position might enable intrafraction corrections in routine stereotactic $\mathrm{RT}$, best applicable if supported by an automated beamhold system in future practice.

\section{Additional file}

Additional file 1: Table S1. Patient and treatment characteristics. Table S2. Descriptive statistics of head motion (3DV), as well as of $x, y$ and $z$ displacements, grouped as measurement cohorts collected within 2-min intervals after treatment start. Figure S1. Patient setup and repeated intrafractional tracking of head movement.

\section{Abbreviations}

3DV: 3D vector; ANOVA: Analysis of variance; CBCT: Cone beam computed tomography; Cl: Confidence interval; IGRT: Image-guided radiotherapy; 
KPS: Karnofsky Performance Scale; $r_{s}$ : Spearman's rank correlation coefficient; RT: Radiotherapy; SD: Standard deviation; $\triangle 3 \mathrm{DV}$ : Change in 3D vector between two consecutive measurements

\section{Acknowledgements}

Not applicable.

\section{Authors' contributions}

$J M$ has made substantial contributions to the conception and design of the work AND acquisition and analysis of data AND interpretation of data AND to the drafting of the work. TS made substantial contributions to the interpretation of data AND to drafting and to substantive revision of the work. RW made substantial contributions to the acquisition and analysis of data. CRA made substantial contributions to the interpretation of data. DV made substantial contributions to the interpretation of data. GG made substantial contributions to the statistical analysis and interpretation of data. PL made substantial contributions to the conception and design of the work. UG made substantial contributions to the interpretation of data. MN-S made substantial contributions to the conception and design of the work AND to drafting and to substantive revision of the work. All authors read and approved the final manuscript.

\section{Funding}

Not applicable.

\section{Availability of data and materials}

The datasets used and analysed during the current study are available from the corresponding author on reasonable request.

\section{Ethics approval and consent to participate}

This study has been approved by the research ethics committee of the Medical University Innsbruck. (reference number: EK 10352019).

\section{Consent for publication}

Not applicable.

\section{Competing interests}

The authors declare that they have no competing interests.

\section{Author details}

'Department of Therapeutic Radiology and Oncology, Medical University of Innsbruck, Anichstraße 35, A-6020 Innsbruck, Austria. ${ }^{2}$ Department of Medical Statistics, Informatics and Health Economics, Medical University of Innsbruck, Innsbruck, Austria.

Received: 21 June 2019 Accepted: 22 November 2019

Published online: 18 December 2019

\section{References}

1. Verellen D, De Ridder M, Storme G. A (short) history of image-guided radiotherapy. Radiother Oncol. 2008;86(1):4-13.

2. Ramakrishna N, Rosca F, Friesen S, et al. A clinical comparison of patient setup and intra-fraction motion using frame-based radiosurgery versus a frameless image-guided radiosurgery system for intracranial lesions. Radiother Oncol. 2010;95(1):109-15.

3. Wurm RE, Erbel S, Schwenkert I, et al. Novalis frameless image-guided noninvasive radiosurgery: initial experience. Neurosurgery. 2008;62(5 Suppl): A11-7 discussion A17-18.

4. Lamba M, Breneman JC, Warnick RE. Evaluation of image-guided positioning for frameless intracranial radiosurgery. Int J Radiat Oncol Biol Phys. 2009;74(3):913-9.

5. Gevaert T, Verellen D, Tournel K, et al. Setup accuracy of the Novalis ExacTrac 6DOF system for frameless radiosurgery. Int J Radiat Oncol Biol Phys. 2012;82(5):1627-35.

6. Solberg TD, Medin PM, Mullins J, Li S. Quality assurance of immobilization and target localization systems for frameless stereotactic cranial and extracranial hypofractionated radiotherapy. Int J Radiat Oncol Biol Phys. 2008;71(1 Suppl):S131-5.

7. Linthout N, Verellen D, Tournel K, Storme G. Six dimensional analysis with daily stereoscopic $x$-ray imaging of intrafraction patient motion in head and neck treatments using five points fixation masks. Med Phys. 2006;33(2):504-13.

8. Spadea MF, Tagaste B, Riboldi M, et al. Intra-fraction setup variability: IR optical localization vs. X-ray imaging in a hypofractionated patient population. Radiat Oncol. 2011;6:38.

9. Blonigen BJ, Steinmetz RD, Levin L, et al. Irradiated volume as a predictor of brain radionecrosis after linear accelerator stereotactic radiosurgery. Int J Radiat Oncol Biol Phys. 2010;77(4):996-1001.

10. Minniti G, Clarke E, Lanzetta G, et al. Stereotactic radiosurgery for brain metastases: analysis of outcome and risk of brain radionecrosis. Radiat Oncol. 2011;6:48.

11. Hellerbach A, Luyken $K$, Hoevels M, et al. Radiotoxicity in robotic radiosurgery: proposing a new quality index for optimizing the treatment planning of brain metastases. Radiat Oncol. 2017;12(1):136.

12. Gevaert T, Steenbeke F, Pellegri L, et al. Evaluation of a dedicated brain metastases treatment planning optimization for radiosurgery: a new treatment paradigm? Radiat Oncol. 2016;11:13.

13. Ruggieri R, Naccarato S, Mazzola R, et al. Linac-based VMAT radiosurgery for multiple brain lesions: comparison between a conventional multi-isocenter approach and a new dedicated mono-isocenter technique. Radiat Oncol. 2018;13(1):38

14. Ohira S, Ueda Y, Akino Y, et al. HyperArc VMAT planning for single and multiple brain metastases stereotactic radiosurgery: a new treatment planning approach. Radiat Oncol. 2018;13(1):13.

15. van Santvoort J, Wiggenraad R, Bos P. Positioning accuracy in stereotactic radiotherapy using a mask system with added vacuum mouth piece and stereoscopic X-ray positioning. Int J Radiat Oncol Biol Phys. 2008;72(1):261-7.

16. Jin JY, Yin FF, Tenn SE, Medin PM, Solberg TD. Use of the BrainLAB ExacTrac Xray 6 D system in image-guided radiotherapy. Med Dosim. 2008;33(2):124-34.

17. Chin LS, Regine WF. Principles and practice of stereotactic radiosurgery. New York: Springer; 2008.

18. Rosenfelder NA, Corsini L, McNair H, et al. Comparison of setup accuracy and intrafraction motion using stereotactic frame versus 3-point thermoplastic mask-based immobilization for fractionated cranial image guided radiation therapy. Pract Radiat Oncol. 2013;3(3):171-9.

19. Kataria T, Gupta D, Karrthick KP, et al. Frame-based radiosurgery: is it relevant in the era of IGRT? Neurol India. 2013;61(3):277-81.

20. Badakhshi $H$, Barelkowski $T$, Wust $P$, et al. Intrafraction variations in linacbased image-guided radiosurgery of intracranial lesions. Cancer Radiother. 2013;17(7):664-7.

21. Lewis BC, Snyder WJ, Kim S, Kim T. Monitoring frequency of intra-fraction patient motion using the ExacTrac system for LINAC-based SRS treatments. J Appl Clin Med Phys. 2018;19(3):58-63.

22. Amelio $D$, Winter $M$, Habermehl $D$, et al. Analysis of inter- and intrafraction accuracy of a commercial thermoplastic mask system used for imageguided particle radiation therapy. J Radiat Res. 2013;54(Suppl 1):i69-76.

23. Wang CW, Lin YC, Tseng HM, et al. Prolonged treatment time deteriorates positioning accuracy for stereotactic radiosurgery. PLoS One. 2015;10(4): e0123359.

24. Accuracy Requirements and Uncertainties in Radiotherapy. Vienna: International Atomic Energy Agency; 2016. https:/www.iaea.org/ publications/10668/accuracy-requirements-and-uncertainties-in-radiotherapy.

\section{Publisher's Note}

Springer Nature remains neutral with regard to jurisdictional claims in published maps and institutional affiliations.

Ready to submit your research? Choose BMC and benefit from:

- fast, convenient online submission

- thorough peer review by experienced researchers in your field

- rapid publication on acceptance

- support for research data, including large and complex data types

- gold Open Access which fosters wider collaboration and increased citations

- maximum visibility for your research: over $100 \mathrm{M}$ website views per year

At BMC, research is always in progress.

Learn more biomedcentral.com/submissions 